\title{
INTEGRATION OF BIOLOGICAL SYSTEMS CONTENT INTO THE PROCESS DYNAMICS AND CONTROL CURRICULUM
}

\author{
Robert S. Parker ${ }^{*, 1}$ Francis J. Doyle III ${ }^{* *}$ \\ Michael A. Henson ${ }^{* * *}$ \\ * Department of Chemical and Petroleum Engineering, \\ University of Pittsburgh, Pittsburgh, PA \\ ** Department of Chemical Engineering, University of \\ California at Santa Barbara, Santa Barbara, CA \\ *** Department of Chemical Engineering, University of \\ Massachusetts at Amherst, Amherst, MA
}

\begin{abstract}
The evolution of the chemical engineering discipline motivates a reevaluation of the process dynamics and control curriculum. A key requirement of future courses is the introduction of theoretical concepts and application examples relevant to emerging areas such as complex biological systems. We outline the critical concepts required to integrate biological content within the traditional framework of process dynamics and control. Our initial experience with three courses is outlined to demonstrate alternative approaches to achieve this integration. The paper concludes with a discussion of open issues which require further attention from the process control and biological systems communities.
\end{abstract}

Keywords: Process control, process dynamics, education, biological systems, biomedical systems, biochemical systems, systems biology.

\section{INTRODUCTION}

The discipline of chemical engineering is evolving, as evidenced by the recent wave of departmental name changes. These have primarily focused on the addition of "bio", such as "biomolecular" (Cornell, Illinois, Notre Dame, Pennsylvania), "biological" (Colorado, Northwestern, Rensselaer Polytechnic Institute, Wisconsin), and "biochemical" (Rutgers). Such name changes reflect both the increasing number of chemical engineering faculty involved in research on biology-oriented topics, and the fact that the percentage of chemical engineering undergraduates going to work for companies in the biotechnology and biomedical sectors has increased from 4.6\% in 1998 to $10.3 \%$ in $2001-02$ (AIChE, 2002).

1 To whom correspondence should be addressed: rparker@ pitt.edu; +1-412-624-7364; 1249 Benedum Hall, Pittsburgh, PA 15261 USA
A series of MIT-organized workshops, supported by the National Science Foundation, examined the current state of undergraduate chemical engineering education and recommended a sweeping set of content and delivery changes (Massachusetts Institute of Technology, 2003). Foremost among the proposed changes were the introduction of biology as a core science, the importance of addressing complexity, and the expanded use of the systems approach. The present discussion focuses on these three elements within the context of the traditional process dynamics and control curriculum.

The dynamics and control course usually taught in the late junior year or senior year is a natural point for including biological systems content along with chemical process material. Due to the focus on key principles rather than specific case studies, biological systems can be integrated seamlessly without detracting from the coverage of more tradi- 
tional applications. The study of complex biological systems often necessitates the integration of concepts from other undergraduate courses such as mass and energy balances, heat and mass transfer and reaction engineering. As a result, the integration of biological systems content has the potential to further expand the role of the dynamics and control course as a capstone experience in the chemical engineering curriculum. Due to their inherent complexity, biological systems offer a rich set of dynamic problems to which well trained chemical engineers can make a significant contribution.

This expanded vision of the system dynamics and control curriculum requires the following difficult issues to be addressed: (1) how can these complex systems be introduced in a meaningful way to undergraduate chemical engineers with little background in biology?; and (2) what changes are required to include biological content without sacrificing the traditional core of process dynamics and control? The objective of this paper is to provide some answers to these questions through the discussion of three courses taught at our respective institutions. The first two examples illustrate the introduction of biological content into the traditional process control course, while the third example focuses on the development of a new course in which the systems approach is applied to a diverse set of biological problems.

\section{INTEGRATION OF BIOLOGICAL SYSTEMS CONTENT}

As with most other courses in the undergraduate chemical engineering curriculum, the process dynamics and control course contains a large span of new material that is covered at a rather brisk pace. To produce students who can apply their knowledge of single- and multi-variable analysis and control in an industrial context is a formidable challenge even when the focus is purely on chemical process systems. The addition of biological content along with the requisite modeling and analysis techniques requires a carefully crafted course to avoid overwhelming students. A possible structure for a semester long system dynamics and control course is illustrated by the syllabus in Table 1 where NL is the number of lectures allotted to the specific topics listed in ALL CAPS. Bold entries represent new topics specific to biological systems. Italicized entries are theoretical topics often considered optional in a traditional control course but which are viewed as important for a biologically oriented course.

The introduction of state-space models and associated analysis tools is essential for the treatment of biological systems due to their complexity (e.g., high order, multivariable, highly nonlinear) often precludes simple Laplace-based analysis. Because students often retain little of the mathematics "learned" in their lower level courses, a few lectures on matrix algebra and linear state-space systems are required to review core
Table 1. Proposed syllabus for a biologically oriented dynamics and control course.

\begin{tabular}{|c|l|}
\hline NL & Topics \\
\hline 4 & $\begin{array}{l}\text { DYNAMIC MODELING } \\
\text { Principles of fundamental modeling; chemical and } \\
\text { biological process examples; introduction to empirical } \\
\text { modeling }\end{array}$ \\
\hline 7 & $\begin{array}{l}\text { LINEAR AND NONLINEAR SYSTEMS ANALYSIS } \\
\text { Matrix algebra and linear state-space systems; linear } \\
\text { systems theory; introduction to nonlinear systems theory; } \\
\text { dynamic simulation; chemical and biological process } \\
\text { examples; introduction to the Laplace transform }\end{array}$ \\
\hline 7 & $\begin{array}{l}\text { FEEDBACK SYSTEMS } \\
\text { Basis principles of feedback; physiological control } \\
\text { systems; homeostasis as a setpoint-free feedback } \\
\text { system; feedback in biochemical reaction networks; } \\
\text { closed-loop response analysis; servo vs. load behavior; } \\
\text { feedback control of chemical process systems; closed- } \\
\text { loop drug delivery }\end{array}$ \\
\hline 8 & $\begin{array}{l}\text { FEEDBACK CONTROL SYNTHESIS } \\
\text { Basic principles of model-based controller design; PID } \\
\text { controller design \& tuning; advanced single-variable } \\
\text { control techniques; multivariable control techniques; } \\
\text { model predictive control; chemical and biological } \\
\text { process examples }\end{array}$ \\
\hline 4 & $\begin{array}{l}\text { ADVANCED TOPICS } \\
\text { Large-scale systems \& plant-wide control; parameter } \\
\text { estimation and experimental design; state estimation; } \\
\text { introduction to systems biology }\end{array}$ \\
\hline
\end{tabular}

material and to ensure that students with deficient backgrounds understand the basic concepts. When combined with the linear systems analysis lecture, this material allows the calculation of eigenvalues to determine stability and matrix rank for the analysis of controllability and observability. The nonlinear systems theory lecture includes the traditional topic of Jacobian linearization as well as introductory coverage of phase plane analysis, multiplicity and bifurcations.

Feedback is a concept easily introduced in the context of biological system examples. Consider the healthy human body versus the insulin-dependent diabetic as a case study. Circulating glucose concentrations are sensed by pancreatic $\beta$-cells. Based on these measurements, $\beta$-cells release an appropriate amount of insulin to maintain the glucose concentration at a physiological reference value. Increasing glucose concentrations lead to increased insulin release and vice versa. The representation of biological control systems using various elements of the traditional block diagram is an appealing framework for presenting biological content. However, this approach can fail to reveal the complexity of the underlying biological processes. In the case of circulating glucose control, a limited degree of complexity can be incorporated by utilizing a nonlinear proportional-derivative controller to represent the pancreatic response to glucose concentration (Nomura et al., 1984). Additional complexity can be added as necessary to allow the application of increasingly advanced closed-loop analysis tools. 
Table 2. Possible case studies for the process dynamics and control course.

\begin{tabular}{l} 
Chemical Processes \\
Continuous and/or fed-batch polymerization reactors; distilla- \\
tion column; continuous pulp digester; paper machine; simple \\
plantwide example (e.g., reactor \& separator); semiconductor \\
process (e.g., lithography); photovoltaic fi lm processing; fuel \\
cell \\
\hline Biotechnological Systems \\
Continuous and/or fed-batch fermentors; yeast energy \\
metabolism; cell stress response (e.g., heat shock); eukaryotic \\
cell cycle; bacterical chemotaxis \\
\hline Biomedical Systems \\
Baroreceptor vagal reflex (blood pressure control system); \\
insulin-dependent diabetic patient (glucose-insulin \\
metabolism/control); circadian rhythm gene regulatory \\
network; anesthesia control; drug delivery for HIV treatment; \\
drug delivery for cancer treatment \\
\hline
\end{tabular}

Throughout the topic sequence in Table 1, a number of examples serve to highlight the breadth of opportunities for application of the theoretical concepts presented in the course. Table 2 provides a list of case studies that could be integrated into a typical dynamics and control class. For each topic where examples are listed in the syllabus, two chemical process and two biological system examples could be used to develop lecture material, in-class exercises and recitation problems. Ideally the biological problems are divided equally between the biotechnology and biomedical lists. However, some material may be illustrated more clearly by selecting both biological examples from the same list. For example, homeostasis is most easily discussed within the context of biomedical processes.

One similarity between the traditional chemical and biological examples listed in Table 2 is the scale on which these systems can be analyzed. Polymerization reactor models can be developed using input-output representations (Parker et al., 2001a) or based on detailed descriptions of the individual polymer particles and their interactions (Immanuel et al., 2003). Analogous models can be developed for microbial fermentors where lumped descriptions of cellular process are provided by unsegregated models (Henson and Seborg, 1992) and detailed descriptions of the individual cells are provided by cell population models (Henson, 2003). A major conclusion of the MIT-organized education workshops was that multi-scale phenomena should be incorporated throughout the undergraduate chemical engineering curriculum (Massachusetts Institute of Technology, 2003). Several of the biological examples listed in Table 2 are well suited for studying system level phenomena characterized by events at small length scales. For example, the baroreceptor vagal reflex can be used to illustrate how activity at the single cell level affects systemic blood pressure control (Doyle III et al., 1997). From the controller design perspective, different types of biological complexity can be examined with the goal of determining the model structure required to synthesize effective controllers for specific bioprocesses. While the introduction of biological systems content is not necessarily required to illustrate these concepts, we feel that an integrated program of chemical and biological examples will reinforce key concepts and demonstrate that these diverse examples are conceptually similar.

\section{SYSTEM DYNAMICS AND CONTROL}

The process control course at the University of Massachusetts has traditionally focused on Laplace transform analysis and chemical process applications. This course usually represents the only extensive exposure to dynamic modeling and feedback control in the undergraduate curriculum. While classical material must be covered for students to gain a working knowledge of the field, advanced control techniques and emerging applications also must be emphasized to reflect current industrial trends. Biological systems were chosen as an appropriate vehicle for introducing the key elements of biological transformations, multi-scale phenomenon and systems level analysis identified in the MIT-sponsored education workshops (Massachusetts Institute of Technology, 2003). Rather than completely change the existing course content, a more conservative approach based on the integration of biological systems and the requisite analysis techniques is being pursued. The short-term objective is to utilize biological system dynamics and feedback to demonstrate the wide applicability of the analytical and computational techniques covered in the course.

The current syllabus for the UMass process control course ( $\mathrm{ChE} \mathrm{446)}$ is shown in Table 3 where new topics introduced in the past year are italicized. The first few weeks are focused on fundamental modeling because undergraduate students typically have little experience formulating dynamic balance equations. A case study approach with traditional chemical process examples and biochemical system examples focusing on yeast metabolism is utilized. A continuous yeast fermentor model is introduced and revisited in lectures and homeworks throughout the semester. Both time domain and Laplace domain analysis techniques receive extensive coverage. An introduction to matrix algebra is necessary since this material is not covered in the required mathematics courses. A major focus is the formulation and stability analysis of linear state-space models.

Engineered and natural feedback systems are introduced in parallel to highlights their common features and unique properties. Multi-scale characteristics of biological control systems are covered by discussing feedback in a yeast metabolic network and the architecture of the baroreceptor reflex. Closed-loop stability is analyzed in both the time and Laplace domains. While most of the material on single-loop controller synthesis is traditional, an introduction to 
Table 3. Syllabus for UMass Course ChE 446: Process Control.

\begin{tabular}{|c|l|}
\hline NL & Topics \\
\hline 5 & $\begin{array}{l}\text { FUNDAMENTAL MODELING } \\
\text { Basic principles; chemical process examples (non- } \\
\text { isothermal chemical reactor; binary flash unit; binary } \\
\text { distillation column); biochemical system examples } \\
\text { (continuous fermentor model; metabolically structured } \\
\text { yeast cell model) }\end{array}$ \\
\hline 7 & $\begin{array}{l}\text { DYNAMIC SYSTEM ANALYSIS } \\
\text { Linear algebra (solution of matrix equations, state-space } \\
\text { models; eigenvalues \& eigenvectors); time domain anal- } \\
\text { ysis (basic stability concepts, linearization of nonlinear } \\
\text { models, linear stability analysis, continuous fermentor } \\
\text { example); Laplace transforms; transfer functions models; } \\
\text { empirical models }\end{array}$ \\
\hline 6 & $\begin{array}{l}\text { FEEDBACK SYSTEMS } \\
\text { Process control systems; biological feedback systems } \\
\text { (engineered versus natural feedback systems, yeast } \\
\text { sulfate assimilation pathway, baroreceptor vagal reflex); } \\
\text { closed-loop transfer functions; closed-loop stability }\end{array}$ \\
\hline 7 & $\begin{array}{l}\text { FEEDBACK CONTROL SYNTHESIS } \\
\text { PID controller tuning; internal model control; time } \\
\text { domain controller design (state feedback, pole place- } \\
\text { ment, model matching, continuous fermentor example); } \\
\text { feedforward control; cascade control }\end{array}$ \\
\hline 5 & $\begin{array}{l}\text { MULTIVARIABLE CONTROL } \\
\text { Control loop interactions; decentralized control; } \\
\text { discrete-time models (discretization of continuous-time } \\
\text { models; convolution models, prediction models); model } \\
\text { predictive control (controller design \& tuning, constraint } \\
\text { handling, real-time optimization; continuous fermentor } \\
\text { example) }\end{array}$ \\
\hline
\end{tabular}

time domain controller design techniques is provided to parallel the Laplace domain methods. The final few weeks are focused on the analysis and design of multivariable control systems. The main emphasis is linear model predictive control since many students entering the oil refining, petrochemical and chemical industries will encounter this technology. The continuous yeast fermentor model is used to illustrate the controller design techniques introduced throughout the course.

To accommodate new material on biological systems and time domain analysis and design techniques, some material typically covered in the process control course had to be deemphasized or virtually eliminated. Topics which received reduced coverage included transfer function models, Laplace domain analysis and design techniques, advanced single loop control strategies and traditional chemical process examples. Frequency domain analysis and design techniques received very limited coverage. While these topics are admittedly valuable, a broader view of dynamic systems and feedback control was deemed to be more important given current trends in the chemical engineering profession.

As part of the ABET mandated evaluation process, students are requested to complete an assessment concerning the course objectives. Four new objectives pertaining to the biological systems content were included in the assessment for the fall of 2003. Each student provided a score ranging from "5" if
Table 4. Student Responses to Biological Systems Content in the UMass Process Control Course.

\begin{tabular}{|c|l|}
\hline Score & Question \\
\hline 3.83 & I can construct a dynamic model of a biological system. \\
\hline 3.78 & $\begin{array}{l}\text { I can perform dynamic system analysis and controller } \\
\text { design in the time domain. }\end{array}$ \\
\hline 3.89 & $\begin{array}{l}\text { I can apply dynamic system analysis techniques to } \\
\text { biological systems to evaluate properties such as } \\
\text { stability. }\end{array}$ \\
\hline 3.83 & $\begin{array}{l}\text { I can describe the relevance of feedback control theory } \\
\text { to biological systems. }\end{array}$ \\
\hline
\end{tabular}

they strongly agreed the objective was achieved to " 1 " if they strongly disagreed the course objective was achieved. Results obtained from the twenty one respondents are summarized in Table 4. The average scores are similar to those obtained for the other course objectives, thereby indicating that the biological content was successfully integrated into the course. Continued improvements in student learning and satisfaction are anticipated as the course content is further developed in the spirit of Table 1.

\section{SYSTEMS-LEVEL BIOMEDICINE}

The University of Pittsburgh Medical Center is a research active hospital system, and the medical doctors collaborate actively with faculty from engineering, business, and the sciences. The biology component in the dynamics and control course (ChE 1034) at Pittsburgh focuses on the analysis of, and controller synthesis for, biomedical systems at the wholeorganism level. By integrating the research activities in modeling and control of diabetic and cancer case studies within the undergraduate class, students are exposed to a component of chemical engineering they might otherwise overlook. This format has resulted in a steady flow of undergraduates interested in undergraduate research, and an increased interest in graduate study.

ChE 1034 is approached from a model-based perspective; approximately half of the course is focused on modeling systems using both fundamental to empirical approaches, in both continuous and sampled-data (discrete) domains. From the fundamental modeling perspective, the students are taught to distinguish pharmacokinetics (the time profile of a drug) from pharmacodynamics (the disease dynamics, effect of the drug on the disease, and toxicity) in much the same way valve dynamics and process output response are captured by separate blocks in a block diagram. In an input-output framework, these two physiologically-motivated effects are indistinguishable, as the pharmacodynamic effect is the observed response. The remainder of the course focuses on the model-based synthesis and analysis of classical and advanced control systems, as in Table 1.

As a case study, consider the insulin-dependent diabetic patient depicted in Figure 1. The fundamental 
model usually covers a recitation session, introducing the students to the key variables of the diabetic patient problem and demonstrating the utility of skills developed elsewhere in the curriculum (e.g. dynamic mass balance with reaction, transport resistance) in the modeling of biomedical problems. As homework or an in-class exercise, the students are asked to reduce this model to a low-order equivalent and justify any assumptions made. The usual result is a model structure containing two differential equations, one each for glucose and insulin, which is conceptually similar to that published in (Bolie, 1961).

The dynamics and control course material is often challenging to the students, who have had a limited introduction to dynamical systems prior to the course. One classical approach to address the challenge of depth of treatment versus limited classroom time is the case study method (Mustoe and Croft, 1999). To provide the students with a consistent example, a collection of case study examples that correspond to the various sections of the curriculum outlined in Table 1 is required. To this end, selections from the insulin-dependent diabetic patient literature have been mapped onto the course outline, as shown in Table 5.

For an expert in the field of diabetic patient modeling and control, this table is fairly straightforward to construct. For the faculty member less familiar with biology, or the diabetic patient problem, the map provides a guide to focused literature reading to quickly bring biomedically motivated problems into the classroom. This aspect is most useful for faculty who are not dynamics and control experts, but who are responsible for teaching the course. This course is challenging for non-experts to teach, and is often rated

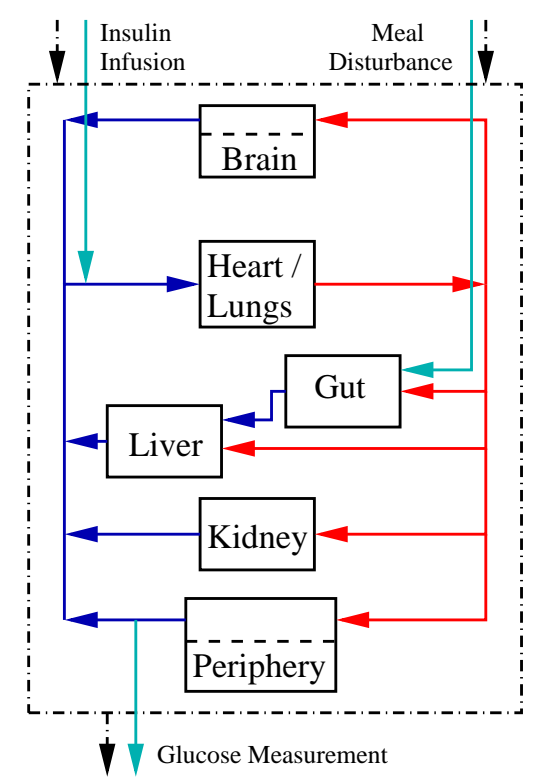

Fig. 1. Open-loop schematic of the diabetic patient. Small solid blocks represent the fundamental model, with manipulated input insulin delivery rate, meal disturbance, and glucose concentration measurement.
Table 5. Integration of sample case study (insulin-dependent diabetic patient) with course outline topics.

\begin{tabular}{|l|}
\hline DATA-DRIVEN MODELING \\
Sorensen FOTD (1985), Bolie two-state linear (1961), \\
Bergman 'Minimal" Model (1981) \\
\hline FIRST PRINCIPLES MODELING \\
Physiologically-based Pharmacokinetic / Pharmacodynamic \\
(Sorensen, 1985; Parker et al., 2000b) \\
\hline LINEAR SYSTEMS ANALYSIS \\
Bolie two-state linear ODEs (1961) \\
\hline LINEAR SYSTEMS ANALYSIS w/ LINEARIZATION \\
Linearize and analyze Bergman 'Minimal" (1981) \\
\hline DYNAMIC SIMULATION \\
All models, including AIDA as a different performance \\
classifi cation (Sorensen, 1985; Bolie, 1961; Bergman et al., \\
1981; Parker et al., 2000b; Lehmann et al., 1994; Agar et \\
al., 2002) \\
\hline FEEDBACK SYSTEMS \\
Glucose-insulin interactions (Bolie, 1961); nonlinear feedback \\
response (Bergman et al., 1981), healthy pancreas response \\
(Sorensen, 1985; Nomura et al., 1984) \\
\hline CLOSED-LOOP ANALYSIS \\
Sorensen healthy patient (Sorensen, 1985) \\
\hline PID CONTROL \\
Controller design from FOTD (Sorensen, 1985), low-order \\
ODEs (Bolie, 1961), and linearized systems and/or effects of \\
nonlinearity (Bergman et al., 1981) \\
\hline ADVANCED CONTROL \\
Feedforward for meal disturbances (Lehmann and Deutsch, \\
1992) and exercise (Lenart and Parker, 2002), with simple \\
(Bolie, 1961; Bergman et al., 1981) or complex (Sorensen, \\
1985) case studies \\
\hline MULTIVARIABLE CONTROL \\
MISO (glucose and insulin inputs; G, I and exercise inputs) \\
(Parker et al.,, 2000a) or MIMO (glucose and insulin control) \\
for a variety of systems (Sorensen, 1985; Bolie, 1961; \\
Bergman et al., 1981) \\
\hline MODEL PREDICTIVE CONTROL \\
Linear MPC in analytical (Bolie, 1961) or data-driven (Parker \\
et al., 1999) forms; MPC with a linearized model (Sorensen, \\
1985; Bergman et al., 1981; Parker et al., 2001b; Lenart and \\
Parker, 2001); nonlinear MPC if desired (Sorensen, 1985; \\
Bergman et al., 1981; Parker et al., , 2001b; Lenart and Parker, \\
2001; Rubb and Parker, 2003) \\
\hline
\end{tabular}

the "least desired teaching assignment" (based on a three-university non-randomized sample of chemical engineering educators). A collection of these papertopic maps, for traditional and biological case studies, would provide those teaching the dynamics and control course a variety of examples tailored to each section of the course.

\section{SYSTEMS BIOLOGY}

In addition to the required dynamics and control course, described in Section 2, there is a demand in many chemical engineering programs for elective courses that facilitate specialization in either systems engineering or biotechnology/biomedical engineering. At UCSB, a new course has been offered in the Spring 2004 quarter, entitled "Engineering Approaches to Systems Biology". The course is taught at a dual-level (seniors and new graduate students), and fulfills the track requirement for both 
systems and biology emphases in the undergraduate chemical engineering program. In addition, it will be cross-listed, in its subsequent offering, with the biomolecular science and engineering (BMSE) program. The current syllabus is listed in Table 6, detailing the topics for a single quarter course (20 lectures of duration 75 minutes).

The course focuses on the emerging problems in systems biology and computational biology. There is a substantial level of effort being invested in these areas in both academia and industry, and the demand for the training of students has increased in proportion. These advances have been facilitated by developments in both computational modeling and high throughput biology - enabling a systematic approach to analyzing complexity in biophysical networks, which was previously untenable. Advances in molecular biology over the past decade have made it possible to probe experimentally the causal relationships between microbiological processes initiated by individual molecules within a cell, and their macroscopic phenotypic effects on cells and organisms. These studies provide increasingly detailed insights into the underlying networks, circuits, and pathways responsible for the basic functionality and robustness of biological systems and create new and exciting opportunities for the development of quantitative and predictive modeling and simulation tools. Model development involves translating identified biological processes into coupled dynamical equations which are amenable to numerical simulation and analysis. These equations describe the interactions between various constituents and the environment, and involve multiple feedback loops, responsible for system regulation, and noise attenuation and amplification.

The discipline of Systems Biology has emerged in response to these challenges (Kitano, 2002), and combines approaches and methods from systems engineering, computational biology, statistics, genomics, molecular biology, biophysics, and other fields. The recurring themes include: (i) integrative viewpoints

Table 6. Syllabus for UCSB Course: ChE 154 - Engineering Approaches to Systems Biology.

\begin{tabular}{|c|l|}
\hline NL & Topics \\
\hline 6 & $\begin{array}{l}\text { CELLULAR REGULATION } \\
\text { Central dogma; genome sequences; genome expression; } \\
\text { genomic circuits; protein, metabolic, signaling networks; } \\
\text { high throughout biological data; biological databases }\end{array}$ \\
\hline 6 & $\begin{array}{l}\text { MATH MODELING AND SYSTEMS ANALYSIS } \\
\text { TOOLS } \\
\text { Modeling strategies; boolean models; nonlinear ODE } \\
\text { models; discrete stochastic models; systems biology } \\
\text { modeling packages; network analysis - robustness, } \\
\text { identifi ability; design of experiment issues }\end{array}$ \\
\hline 6 & $\begin{array}{l}\text { BIOSYSTEMS CASE STUDIES } \\
\text { Bacterial chemotaxis; lambda phage; circadian rhythm; } \\
\text { caspase signaling cascade; pap pili phase variation }\end{array}$ \\
\hline 2 & $\begin{array}{l}\text { COURSE PROJECTS } \\
\text { Midterm progress reports; fi nal presentations }\end{array}$ \\
\hline
\end{tabular}

towards unraveling complex dynamical systems, and (ii) tight iterations between experiments, modeling, and hypothesis generation.

In response, there have been a number of courses introduced in a variety of departments across the country that address elements of systems biology and computational biology. These have been targeted at both undergraduate and graduate audiences, and in some cases involve continuing education participants from industry. The balance of topics in the syllabus in Table 6 is approximately one third on basic cellular regulation, one third on applications of systems engineering tools to biological problems, and one third on detailed case studies to illustrate current methodologies and future challenges. Although the UCSB curriculum is based on quarters, the same general template could be extended to a semester-long course, without significant modification.

Assignments for this course consist of short homework problems, primarily at the beginning of the course, and a major course project. The project entails a midterm progress report, a final presentation, and a written report. The case study offers a mechanism to tailor the course to a diverse student population seniors work on teams with a reduced scope, while graduate students work as individuals on a more detailed project.

\section{OPEN ISSUES IN UPDATING THE PROCESS DYNAMICS AND CONTROL COURSE}

\subsection{Reducing the Emphasis on the Laplace Domain}

Traditional process control courses emphasize the Laplace transform for solution of dynamic models as well as for the analysis of open- and closedloop systems. In the coverage of multivariable systems, a common approach is to construct singleinput, single-output (SISO) transfer function models for each input-output pair and then combine the SISO models into a transfer function matrix. While classical analysis may be facilitated by using Laplace domain system representations, inherent limitations (e.g. linear system, deviation variables, "small" input-output dimension) severely restrict the utility of this approach for complex systems commonly encountered in biological applications. Biological systems are inherently nonlinear with phenomena ranging from protein interactions in gene regulatory networks to adaptation in systemic reflexes. Furthermore, modeling of biological systems at resolutions below the macroscopic scale often leads to high state dimension (Henson, 2003; Parker et al., 2000b).

$>$ From this perspective, complex dynamic systems are most effectively addressed in the time domain. Nonlinear analysis techniques can be introduced explicitly in the time domain (see Section 3), thereby exposing students to theoretical concepts and analysis 
tools with wider applicability than Laplace domain methods. Moreover, the formulation of large-scale system models (in terms of state and/or input-output dimensions) is more readily performed in the time domain via conservation equations. Consequently, the syllabus in Table 1 focuses on the derivation and analysis of linear and nonlinear state-space systems. Connections with the corresponding Laplace domain concepts can be introduced as necessary (e.g. stability via eigenvalues vs. poles).

As is evident from Table 1, frequency domain analysis and design techniques have been effectively removed from the proposed curriculum. While we do not dispute the potential value of these methods, our experience is that this material is difficult for most students to understand and apply. Because frequency domain analysis often starts with the substitution of $s=j \omega$ into the transfer function model, the removal of frequency domain material is consistent with the reduced emphasis on the Laplace transform. On the other hand, the syllabus in Table 1 is sufficiently flexible that limited coverage of frequency domain methods at the expense of other topics is possible. For instructors interested in including these tools, brief coverage of the fast Fourier transform (FFT) and pulse testing may be represent a suitable compromise.

The Laplace transform is particularly useful for the analysis of systems with time delay and/or zero dynamics. While numerical simulation of these systems is straightforward, analysis in the time domain is more difficult. Computational tools such as the FFT can be employed, but such methods are not well suited for use in conventional written exams. Analytical treatment of zeros in the time domain is more involved than the corresponding Laplace domain methods for SISO linear systems.

Time domain analysis of transportation and measurement delays is most conveniently performed using a discretized framework. Linear state-space models without delay can be constructed by performing state augmentation and using shift matrices. While this approach can lead to potentially large state dimensions, properly formulated exam problems can partially mitigate this problem. Multiple delays and delays between blocks in a closed-loop system produce more complicated state-space models that are problematic in the context of written exams. Consequently, evaluating student understanding of this material can be challenging. Two possible approaches are: (i) prepare challenging problems and conduct exams in computational classrooms where students have access to the tools necessary to complete the analysis; and (2) construct two-part problems with the first part focuses on problem formulation and the second part addresses the analysis problem on a related but simplified problem.

\subsection{Multivariable Control}

While most traditional courses treat multivariable systems as a straightforward extension of SISO systems, a more comprehensive approach which addresses the unique challenges of multivariable controller design is required. A formal introduction to decentralized control would support the "systems viewpoint" when performing control relevant analysis of multivariable processes - a set of optimal SISO feedback loops generally does not result in overall system optimality. While coverage of this material is relatively straightforward, the vast majority of multivariable systems are suboptimally controlled when single-loop controller design techniques are employed. Furthermore, students working in the chemical process industries will invariably encounter model predictive control applications. While comprehensive treatment of model predictive control is beyond the scope of this course, students need to develop a working knowledge of this high performance control technology. As outlined in the course syllabus in Table 3, the introduction of this topic necessitates limited coverage of discrete-time models and real-time optimization.

\subsection{Robustness}

A critical topic in the analysis of both process control systems and biological regulation is robustness. The remarkable levels of robust performance attained in nature is enviable from an engineering perspective and is not a well understood issue in biology. The importance of robustness in understanding disease states, as well as evolution and development, motivate its incorporation in the dynamics and control curriculum. Of course, a detailed theoretical treatment, such as that provided in (Skogestad and Postlethwaite, 1996), is beyond the scope of a typical undergraduate course. However, key concepts of robustness can be emphasized using simple tools such as sensitivity analysis - effectively capturing the gains from uncertain elements in a system to the controlled output or performance measure. Analysis can include the closed-loop strategies that have been adopted in nature to deal with robustness, such as redundancy, feedback, filtering and modular protocols.

\subsection{Teaching Control for Non-Expert Faculty}

Our experience indicates that the process dynamics and control class is not a popular choice as a teaching assignment among non-experts in the field. The lack of interest is due to a variety of issues, including the mathematical complexity of the material and the significant focus on feedback controller synthesis. An additional concern is that the material is challenging to students, who have had a limited introduction to dynamical systems prior to this course. The syllabus in Table 1 represents a significant departure from the 
traditional controller synthesis dominated course to a more balanced presentation of system dynamics and feedback.

A notable benefit of the proposed syllabus is the degree of potential customization. While our focus has been on the introduction of biological systems content, the treatment of other application areas such as advanced materials or microelectronics can be accomplished in a similar manner. This flexibility provides an excellent opportunity for instructors to integrate their research interests into the course. The three courses described in Sections 3-5 were heavily influenced by the work performed in the corresponding research groups. Possible benefits of such integration include: (i) increasing the diversity of application examples by encouraging non-experts to teach the course; and (ii) introducing students to cutting edge engineering research which influences their perception of the field and may affect their future career directions.

Experts in process dynamics and control can contribute to the development of instructional materials in a variety of ways. The construction of extended case studies such as Table 5 for different applications (e.g. electronic materials) would ease the burden on non-experts to incorporate novel examples into the curriculum. Software tools such as the Process Control Modules (Doyle III et al., 2000) and Java-based Control Modules (Yang and Lee, 2002) are well suited for introducing traditional concepts and applications. New software tools are needed to increase the exposure of chemical engineering undergraduates to biological complexity and to allow the application of the theoretical concepts introduced in the course to representative biological systems. Ongoing efforts, such as those organized by MIT and the CACHE organization, are focused on the development and refinement of biologically-relevant systems courses. A task force headed by the second author of this paper is currently working on course revisions as well as software module design as a means to integrate biological content throughout the chemical engineering curriculum. More details will be made available at www. cache. org.

\section{REFERENCES}

Agar, B. U., G. Birol and A. Cinar (2002). Virtual experiments for controlling blood glucose level in type I diabetes. In: Proc. Second Joint EMBS/BMES Conf.. p. 2609.

AIChE (2002). 2001-2002 initial placement of chemical engineering graduates. http://www.aiche.org/careerservices/trends/ placement.htm.

Bergman, R.N., L.S. Phillips and C. Cobelli (1981). Physiologic evaluation of factors controlling glucose tolerance in man. $J$. Clin. Invest. 68, 1456-1467.

Bolie, V. W. (1961). Coeffi cients of normal blood glucose regulation. J. Appl. Physiol. 16, 783-788.

Doyle III, F. J., M. A. Henson, B. A. Ogunnaike, J. S. Schwaber and I. Rybak (1997). Neuronal modeling of the baroreceptor reflex with applications in process modeling and control. In: Neural Networks for Control (D.L. Elliott, Ed.). Springer Verlag.
Doyle III, F. J., R. S. Parker and E. P. Gatzke (2000). Process Control Modules: A Software Laboratory for Control Design. Prentice Hall International Series in the Physical and Chemical Engineering Sciences. PH PTR. Upper Saddle River, NJ 07458.

Henson, M. A. (2003). Dynamic modeling of microbial cell populations. Current Opinion in Biotechnology 14, 460-467.

Henson, M. A. and D. E. Seborg (1992). Nonlinear control strategies for continuous fermentors. Chem. Eng. Sci. 47, 821-835.

Immanuel, C. D., C. F. Cordeiro, S. S. Sundaram, E. S. Meadows, T. J. Crowley and F. J. Doyle III (2003). Modeling of particle size distribution in emulsion co-polymerization: Comparison with experimental data and parametric sensitivity studies. Comp. Chem. Eng. 26, 1133-1152.

Kitano, H. (2002). Systems biology: A brief overview. Science 295, 1662-1664.

Lehmann, E. D., T. Deutsch, E. R. Carson and P. H. Sonksen (1994). AIDA: An interactive diabetes advisor. Comp. Meth. Prog. Biomed. 41, 183-203.

Lehmann, E.D. and T. Deutsch (1992). A physiological model of glucose-insulin interaction in type 1 diabetes mellitus. $J$. Biomed. Eng. 14, 235-242.

Lenart, P. J. and R. S. Parker (2001). Glucose control during exercise in type I diabetic patients. Proceedings of the Topical Conference on Bioinformatics and Genomics. AIChE Annual Meeting.

Lenart, P. J. and R. S. Parker (2002). Modeling exercise effects in type I diabetic patients. Proceedings of the 15th IFAC World Congress on Automatic Control. Barcelona, Spain.

Massachusetts Institute of Technology (2003). Frontiers in chemical engineering education initiative. http://web.mit.edu/checurriculum/.

Mustoe, L. R. and A. C. Croft (1999). Motivating engineering students by using modern case studies. International Journal of Engineering Education 15, 469-476.

Nomura, M., M. Shichiri, R. Kawamori, Y. Yamasaki, N. Iwama and H. Abe (1984). A mathematical insulin-secretion model and its validation in isolated rat pancreatic islets perfusion. Comput. Biomed. Res. 17, 570-579.

Parker, R. S., D. Heemstra, F. J. Doyle III, R. K. Pearson and B. A. Ogunnaike (2001a). The identification of nonlinear models for process control using tailored "plant-friendly" input sequences. J. Proc. Control 11, Sp. Issue SI, 237-250.

Parker, R. S., E. P. Gatzke and F. J. Doyle III (2000a). Advanced model predictive control (MPC) for type I diabetic patient blood glucose control. In: Proc. American Control Conf.. Vol. 5. pp. 3483-3487.

Parker, R. S., F. J. Doyle III and N. A. Peppas (2001b). The intravenous route to blood glucose control. IEEE Eng. Med. Biol. 20, 65-73.

Parker, R. S., F. J. Doyle III and N. A. Peppas (1999). A modelbased algorithm for blood glucose control in type I diabetic patients. IEEE Trans. Biomed. Eng. 46(2), 148-157.

Parker, R. S., J. H. Ward, N. A. Peppas and F. J. Doyle III (2000b). Robust $\mathrm{H}_{\infty}$ glucose control in diabetes using a physiological model. AIChE Journal 46, 2537-2549.

Rubb, J. D. and R. S. Parker (2003). Glucose control in type I diabetic patients: A Volterra model-based approach. In: Proc. ADCHEM 2003. Vol. in press.

Skogestad, S. and I. Postlethwaite (1996). Multivariable Feedback Control. John Wiley \& Sons, New York, NY.

Sorensen, J.T. (1985). A Physiologic Model of Glucose Metabolism in Man and its Use to Design and Assess Improved Insulin Therapies for Diabetes. PhD thesis. Department of Chemical Engineering, MIT.

Yang, D. R. and J. H. Lee (2002). Process control education software using Java applet. AIChE Annual Meeting. http://dot.che.gatech.edu/Information/research/issicl/che4400/ javamodule.html. 